\title{
Synthesis of Optimized Asymmetrical Sum Patterns Using Conventional Method
}

\author{
M. Satya Anuradha, ${ }^{1}$ Dr P. V.Sridevi ${ }^{2}$, Prof G. S. N.Raju ${ }^{3}$ \\ ${ }^{1}$ (Sr Assistant Professor, ECE dept, AUCE (A), Andhra University, INDIA) \\ ${ }^{2}$ (Associate Professor, Andhra University, INDIA) \\ ${ }^{3}$ (Vice Chancellor, Andhra University, INDIA)
}

\begin{abstract}
Sum patterns find applications in Radar for searching and ranging of the targets. A sum pattern with low sidelobe level is a desirable feature in these applications, in order to reduce EMI problems. Sum patterns with Asymmetrical sidelobe topography are considered, in applications where only certain angular regions of pattern are required to have low sidelobe level. Asymmetrical pattern characteristics can have lower beam widths for given design specifications as compared to symmetrical patterns. In view of this, a conventional method of synthesis is carried out in this paper, to produce asymmetrical sidelobe level patterns using discrete arrays. The effect of beam scanning on the pattern behavior is also analyzed for the above synthesized patterns.
\end{abstract}

Keywords: sum pattern, asymmetrical sidelobe level, beam width, complex excitation weights, and discrete array.

\section{Introduction}

A common pattern requirement is high directivity and low sidelobe level for Radar, communication and mapping applications. The main beam may be fixed at broad side or at some angle. Usually the radiation pattern of a single element is relatively wide and provides a very low value of directivity. In many applications it is necessary to design antennas with very directive characteristics to meet the demands of long distance communication. This can only be accomplished by increasing the electrical size of the antenna. Enlarging the dimensions of single element leads to more directive characteristics, which sometimes increases the system complexity. Another way to increase the directivity without necessarily increasing the size of the element is to form an assembly of radiating elements in electrical and geometrical configuration [1].

There are at least five controls that can be used to control the overall pattern of the antenna. They are number of elements in the array, the geometrical configuration of the overall array, the excitation of the individual elements, relative displacement and the radiation patterns of the individual elements. A linear array consists of equally spaced elemental radiators [2], laid out in a straight line, the sum pattern is characterized by a single narrow main lobe and a set of side lobes. For most of the applications the sum pattern should possess narrow mainlobe and very low sidelobes.

In Radar and Communication applications, sum patterns with low side lobe levels are useful in order to have low beam widths. In some radar applications sum patterns with asymmetrical side lobes provide a system advantage [2]. The sum patterns with arbitrary side lobe topology are useful in applications where undesired signals are coming from a limited region of space, permitting the sidelobes to be higher elsewhere, results in a narrow main beam and more directivity from the same aperture [3]. Frequently antennas operate in an environment with several targets or interfering objects present. This may lead to ambiguous or false system response. A rather substantial error in the system response may be due to the contributions from the pattern sidelobes. Therefore it is desirable to keep the sidelobe level as low as possible. There exist several methods for designing line sources and uniformly spaced arrays which have lower sidelobe and narrow beam widths.

However there is only specific angular region over which the antenna response must be very low. Reduction of all sidelobes to some very low level is possible in theory, but leads to wider main beam width and may be difficult to realize in practice. It is therefore important to be able to synthesize patterns with very low side lobes over one or more specified sectors of the pattern. Hyneman prescribed one method for achieving control over the near in sidelobe envelope function for line sources.

Sum patterns can be generated with standard distributions and designed distributions. Instead of equal currents and equal phases, symmetrical taper distributions can be utilized, but the tapered distribution suffers the penalty of some increase in beam width to the first null. However, this sacrifice is benefited by a compensatory advantage of secondary minimum or first sidelobe lower than it was in the case of the uniform current distribution. The important conclusion is that the SLL can be controlled by tapering the array excitations, at some cost in beamwidth. The angular excitation of the main beam in a sum pattern is inversely related to the length of the array and for a given array length the main beam broadens as the sidelobe level is lowered. The 
design specifications of synthesis problem include desired width of the sum pattern as well as the sidelobe level.

The designer determines the requisite current distribution, array length and the spacing of the elements

Design techniques for synthesis of line source aperture distribution that yield antenna radiation pattern with narrow main beam and symmetrical low sodelobes have been highlighted by the work of Dolph and Taylor.

Dolph utilized the feature that Chebyshev functions consists of a number of unitary oscillations,

followed by a hyperbolic rise, in designing linear arrays that would produce antenna patterns replicating the Chebyshev characteristics. He has shown that for discrete array of linear radiators, spaced a half wave length apart, an optimality condition exists in that Dolph pattern provide a minimum beam width for given sidelobe level. Taylor demonstrated that the continuum limit of the Dolph aperture distribution is physically un realizable, but can be approximated quite satisfactorily by a distribution that corresponds to a radiation pattern with a narrow main beam and symmetric sidelobes, a specified number of these sidelobes on each side of the main beam (extending to the limit of the visible region, if desired) can be designed to be at essentially the same level, with the farther out sidelobes decaying in height. The Taylor pattern is representable as a canonical product of factors whose roots are the zeros of the pattern, however both Dolph and Chebychev distribution provide patterns with symmetric sidelobes, and in some applications this is neither a requirement nor even desirable. Typical of such applications is the situation in which the main beam to point to prescribed degrees above the horizon and the sidelobes between the horizon and main beam are to be suppressed to lower levels to minimize ground clutter.

If a Dolph or taylor is employed, the sidelobes between the main beam and zenith will also be suppressed to the same level. However it might be that, in a case where the upper region of sidelobes need not be suppressed that deeply, if the sidelobes are not suppressed that much, there would be a narrowing of the main beam and an increase in gain relative to the symmetric case with suppressed all sidelobes to the same level. It is this feature that makes the idea of a pattern with asymmetric sidelobes appealing in such situations. Some of the system applications also require imbalances in sidelobes, like marine radar applications. A simple modification of the Taylor distribution has been found to give designer control over the asymmetric levels of the sidelobes on the two sides of the main beam.

\section{Formulation}

The continuous line source being represented by magnetic currents, the element factors and a common array factor, being an integral form by considering the general array factor

$$
\delta(\theta)=\int_{-a}^{a} g(x) e^{j k x \sin (\theta)} d x
$$

$g(x)$ is the required amplitude distribution

$\delta(\theta)$ is the desired pattern

$k=\frac{2 \pi}{\lambda}$

$g(\zeta)=K e^{-j \beta x}$ with $K$ and $\beta$ constants, as a special case the aperture has uniform amplitude, uniform progressive phase, results in a sum pattern with symmetrical side lobes whose field heights trail off as $\mathrm{u}^{-1}$, the pair of side lobes closest is being down $13.5 \mathrm{~dB}$. One could depress the innermost side lobes on each side of the main beam to a common height, meanwhile leaving alone all the side lobes that are further out. An approximation to such patterns can be constructed in the following way: Select an integer $\bar{n}$ and say that for $|\boldsymbol{u}| \geq \bar{n}$, the nulls of the new pattern are to occur at integral values of $u$. But the next pair of nulls toward the main beam will need to occur at $\boldsymbol{u}= \pm \boldsymbol{u}_{\bar{n}_{-1}}$, where $\left|\boldsymbol{u}_{\bar{n}-1}\right|=\overline{\boldsymbol{n}}-\mathbf{1}$, in order to depress the intervening side lobes somewhat. Similarly, the penultimate pair of nulls needs to be shifted to $\boldsymbol{u}=\bar{n}-2$ where $\left|\boldsymbol{u}_{\bar{n}-2}\right|=\bar{n}-2$, and so on. The function that expresses this new pattern is the Taylor pattern that can be expressed in the form of 


$$
\delta(\boldsymbol{u}, \boldsymbol{A}, \bar{n})=\frac{\sin \pi u}{\pi u} \prod_{n=1}^{n-1}\left\{1-\frac{u^{2}}{\sigma^{2}\left[A^{2}+\left(n-\frac{1}{2}\right)^{2}\right]}\right\} \frac{1}{\prod_{n=1}^{n-1} 1-\frac{u^{2}}{n^{2}}}
$$

Which remove the inner most $\bar{n}-1$ pair of nulls from the original $\frac{\operatorname{Sin} \pi u}{\pi u}$ pattern and replace them with new pairs at modified position $\pm u_{n}$ the new null positions should be determined from the formula

$$
u_{n}=\bar{n}\left[\frac{A^{2}+(n-1 / 2)^{2}}{A^{2}+(\bar{n}-1 / 2)^{2}}\right]^{1 / 2}
$$

$\boldsymbol{A}$ is a measure of the side lobe level (SLL) in that $\cosh \pi A=\mathrm{b}$, with $20 \log ,, b=\mathrm{SLL}$.

With a Taylor pattern defined by [2] and [3], it becomes a simple matter to find the corresponding aperture distribution from [1].

Let $g(x)=h(x) e^{-j \beta x}$ be the excitation function, with $h(x)$ represented by the Fourier series

$$
h(x)=\sum_{m=0}^{\infty} B_{m} \frac{\cos m \pi x}{a}
$$

However, [1] indicates that $\delta(m)=0$ for $m \geq \bar{n}$, so the Fourier series truncates, and thus the continuous aperture distribution is given by

$$
g(x)=\frac{e^{-j \beta x}}{2 a}\left[\delta(0)+2 \sum_{m=1}^{\bar{n}-1} \delta(m) \cos m \pi x / a\right]
$$

\section{Modified Taylor Patterns}

Optimum designs of sum patterns often call for the maximum directivity (minimum beam width) from a line source of specified length, subject to some specification on the side lobe level. However, all directions in space may not be equally important, so far as side lobe suppression is concerned. Since every side lobe that is suppressed costs something in beam broadening, Taylor patterns (which arise when equal importance is attached to all directions) may not be optimum in some applications one is led to consider designs which permit high side lobes in unimportant regions, while maintaining low side lobes in critical regions, that is, patterns with arbitrary side lobe topography.

A perturbation procedure may be used to modify a Taylor pattern so that all the side lobes have individually arbitrary heights. To begin with, expressing the Taylor pattern of [1] in the more general form

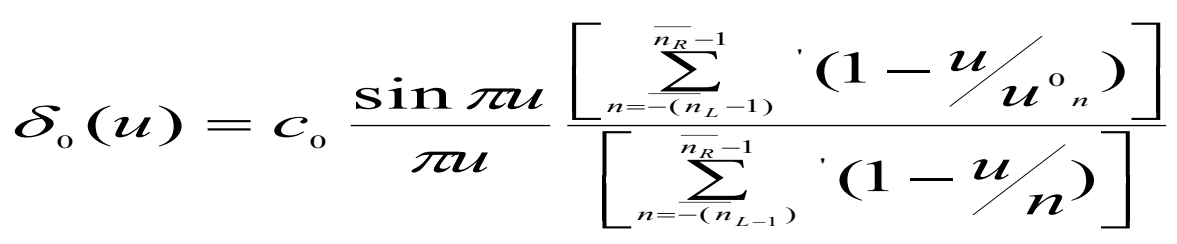

The subscripts $\mathrm{R}$ and $\mathrm{L}$ in [5] are used to identify the right side and the left side of the pattern. As generalized, the root positions are given by 


$$
\begin{aligned}
& u_{n}{ }^{\circ}=\overline{n_{R}}\left[\frac{A_{R}{ }^{2}+(n-1 / 2)^{2}}{A_{R}{ }^{2}+\left(\overline{n_{R}}-1 / 2\right)^{2}}\right]^{1 / 2} \\
& n=1,2, \ldots \ldots \ldots \ldots, \bar{n}_{R}-1 \\
& u_{n}{ }^{0}=-\overline{n_{L}}\left[\frac{A_{L}{ }^{2}+(n-1 / 2)^{2}}{A_{L}{ }^{2}+\left(\overline{n_{L}}-1 / 2\right)^{2}}\right] \quad n=-1-, 2, \ldots \ldots \ldots .,-\bar{n}_{L}-1
\end{aligned}
$$

In (6) through (8), $\overline{\boldsymbol{n}}_{L}$ and $\overline{\boldsymbol{n}}_{R}$, are positive integers that denote the transition roots on the two sides of the main beam. The side lobe level parameters on the two sides of the pattern are, $A_{L}$ and $A_{R}$. The prime on each product sign in (6) indicates that the factor for which $n=0$ has been excluded. Specifically, this pattern has the advantage that the beam width is narrower and the directivity is higher than one finds in a symmetrical Taylor. The aperture distributions corresponding to these modified Taylor patterns can be expressed in the Fourier form.

$$
g(x)=\frac{e^{-j \beta x}}{2 a}\left[\sum_{m=-\left(\overline{n_{L}}-1\right)}^{\overline{n_{R}}-1} \delta_{\mathrm{O}}(m) e^{-j m \pi x / a}\right]
$$

The above equation gives the excitation function for continuous line source antenna. Magnitude and phase of the above equation are used as excitation amplitude and phase functions to get the asymmetrical sidelobe level patterns. The excitation weights for discrete array are obtained by taking samples at the element positions of continuous excitation functions [5]. For discrete array the pattern function is given by the following equation.

$\sum_{n=-N}^{n=N} A_{n} \quad e^{j n\left(k d u+\varphi\left(x_{n}\right)\right)}$

\section{RESULTS}

The figures 1 to 8 indicate the asymmetrical sidelobe patterns and their excitation amplitude and phase functions. The results were presented for an array length of 14 and 12 . The sidelobe level of $15 / 45 \mathrm{~dB}$, are considered for $\overline{n_{L}}=4, \overline{n_{R}}=6$. The second set of parameters considered are SLL $=35 / 15 \mathrm{~dB}, \overline{n_{L}}=6, \overline{n_{R}}=8$. Tables-1 gives the synthesized root values for $\mathrm{SLL}=15 / 45 \mathrm{~dB}$ and Table- 2 indicates the excitation weights for $\mathrm{SLL}=35 / 15 \mathrm{~dB}, \mathrm{~N}=24$.

Table -1 Root values for $\mathrm{SLL}=15 / 45 \mathrm{~dB}$

\begin{tabular}{|c|c|c|c|c|c|c|c|c|c|}
\hline S.No & 1 & 2 & 3 & 4 & 5 & 6 & 7 & 8 \\
\hline $\mathrm{n}$ & -3 & -2 & -1 & 0 & 1 & 2 & 3 & 4 \\
\hline $\begin{array}{c}\text { Root } \\
\text { value }\end{array}$ & -0.01411 & 0.025366 & 0.00916 & 1.04 & 0.323701 & $-5.7 \mathrm{E}-05$ & 0.004154 & -0.0019 & 0.000634 \\
\hline
\end{tabular}


Table -2 Excitation weights for SLL $=35 / 15 \mathrm{~dB}, \mathrm{~N}=24$

\begin{tabular}{|c|c|c|c|}
\hline S. No & Element position & Amplitude & Phase in Degrees \\
\hline 1 & -0.95833 & 0.392641 & -17.1171 \\
\hline 2 & -0.875 & 0.409644 & -41.2624 \\
\hline 3 & -0.79167 & 0.424224 & -46.1559 \\
\hline 4 & -0.70833 & 0.487753 & -40.1399 \\
\hline 5 & -0.625 & 0.586954 & -35.6356 \\
\hline 6 & -0.54167 & 0.673079 & -31.9028 \\
\hline 7 & -0.45833 & 0.754014 & -27.1031 \\
\hline 8 & -0.375 & 0.83343 & -22.423 \\
\hline 9 & -0.29167 & 0.896392 & -17.7307 \\
\hline 10 & -0.20833 & 0.946465 & -12.6159 \\
\hline 11 & -0.125 & 0.983803 & -7.62258 \\
\hline 12 & -0.04167 & 1 & -2.61256 \\
\hline 13 & 0.041667 & 1 & 2.612562 \\
\hline 14 & 0.125 & 0.983803 & 7.622583 \\
\hline 15 & 0.208333 & 0.946465 & 12.61593 \\
\hline 16 & 0.291667 & 0.896392 & 17.73068 \\
\hline 17 & 0.375 & 0.83343 & 22.42299 \\
\hline 18 & 0.458333 & 0.754014 & 27.10311 \\
\hline 19 & 0.541667 & 0.673079 & 31.90283 \\
\hline 20 & 0.625 & 0.586954 & 35.63556 \\
\hline 21 & 0.708333 & 0.487753 & 40.13985 \\
\hline 22 & 0.791667 & 0.424224 & 46.15591 \\
\hline 23 & 0.875 & 0.409644 & 41.26238 \\
\hline 24 & 0.958333 & 0.392641 & 17.11709 \\
\hline
\end{tabular}

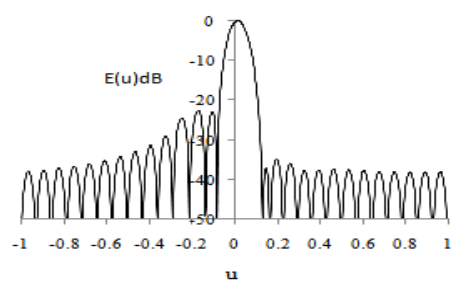

Fig 1. Synthesized Unsymmetrical pattern having $N=28$. SLL $=15 / 45 \mathrm{~dB}$.

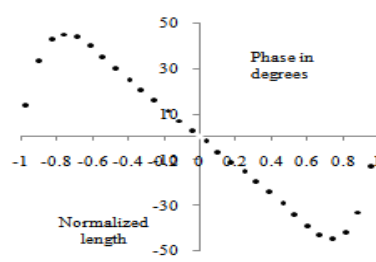

Fig 3. Phase excitation weights for unsymmetrical pattern having SLI $=15 / 45 \mathrm{~dB}$

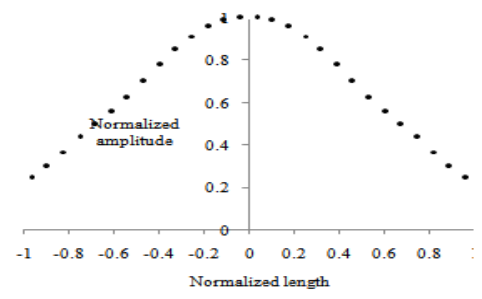

Fig 2. Amplitude excitation weights for unsymmetrical pattern having SLL $=15 / 45 \mathrm{~dB}$.

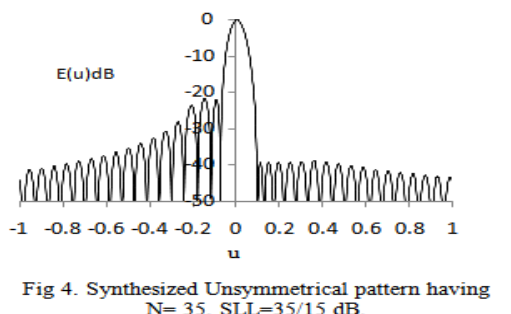

$\mathrm{N}=35, \mathrm{SLL}=35 / 15 \mathrm{~dB}$ 


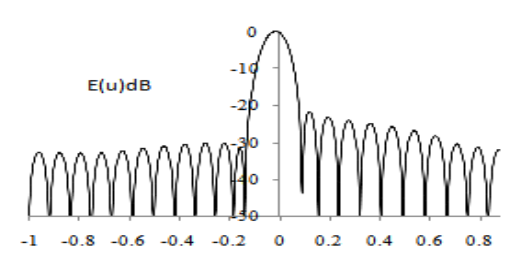
Fig 5. Synthesized Unsymmetrical pattern

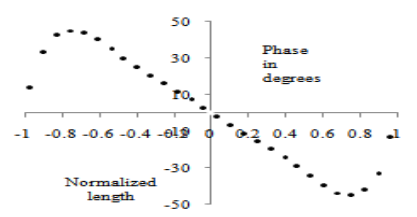

Fig 7. Phase excitation weights for
unsymmetrical pattern having SLL $=35 / 15 \mathrm{~dB}$

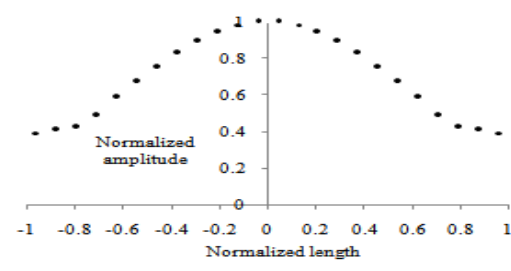

Fig 6. Amplitude excitation weights for unsymmetrical pattern having $S L L=35 / 15 \mathrm{~dB}$

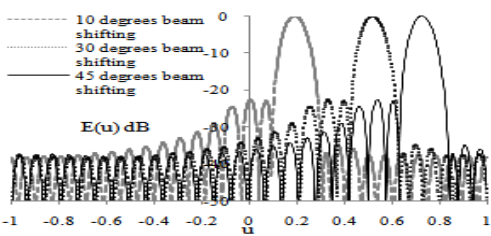

Fig 8. Synthesized Unsymmetrical pattern having $\mathrm{N}=50$, Phase shift of $10,30,45$ Degrees

\section{Conclusion}

This paper discusses conventional method to synthesize asymmetrical sum patterns. Modified Taylor method of synthesis is used to obtain the desired patterns. The excitation phase and amplitude are calculated for continuous line source, and these excitations are sampled at the element positions of the discrete array provided the array length is large. Synthesized asymmetrical SLL ratio is differing from the desired SLL by $\leq 5 \mathrm{~dB}$ level. The synthesized excitation amplitude is even symmetric about the origin and phase excitation is odd symmetric about the origin. This analytical method of synthesis requires more mathematical analysis, sometimes it can be solved by optimization techniques.

\section{Reference}

[1] Richard F. Hyneman, "A Technique for the Synthesis of Line-Source Antenna Patterns Having Specified Sidelobe Behavior", IEEE Transactions on Antennas and Propagation, Vol .AP.16, No. 4, pp. 430-435, July 1968.

[2] 2.R.S. ELLIOTT, "Design of Line Source Antennas for Narrow Beamwidth and Asymmetric Low Sidelobes", IEEE Transaction on antennas and propagation, pp. 100-107, January 1975.

\section{Text Books.}

[3] Robert S.Elliott, "Antenna Theory and Design", published by "A Jhon Wiley \& Sons, INC".

[4]. G. S. N. Raju, "Antennas and wave propagation”, Pearson Education, 2005. 